\title{
Average transverse momentum of hadrons in proton-nucleus collisions in the wounded nucleon model
}

\author{
Adam Bzdak ${ }^{\mathrm{a}}$, Vladimir Skokov ${ }^{\mathrm{b}}$ \\ ${ }^{a}$ RIKEN BNL Research Center, Brookhaven National Laboratory, Upton, NY 11973, USA \\ ${ }^{b}$ Department of Physics, Brookhaven National Laboratory, Upton, NY 11973, USA
}

\begin{abstract}
In the wounded nucleon model, a proton-nucleus $(\mathrm{p}+\mathrm{A})$ collision is a superposition of independent nucleon-nucleon collisions. We use this model to calculate the average transverse momentum of pions, kaons and protons in high energy $\mathrm{p}+\mathrm{A}$ collisions. For the same number of produced particles, because the number of participants differs, in the wounded nucleon model the transverse momentum of hadrons can differ between $\mathrm{p}+\mathrm{p}$ and $\mathrm{p}+\mathrm{A}$ collisions. In this model we find that the average transverse momentum in high multiplicity $\mathrm{p}+\mathrm{A}$ collisions depends weakly on the number of produced particles, and underestimates the preliminary experimental data at the LHC. The difference is small for pions and greater for kaons and protons. The magnitude of this difference is consistent with hydrodynamic expectations.
\end{abstract}

\section{Introduction}

Recent results on the two-particle correlation function in $\mathrm{p}+\mathrm{p}$ [1], $\mathrm{p}+\mathrm{Pb}[2$, $3,4,5]$ and $\mathrm{Pb}+\mathrm{Pb}[6,5]$ collisions at the $\mathrm{LHC}$, and in $\mathrm{d}+\mathrm{Au}[7]$ collisions at RHIC demonstrated the existence of the long-range correlations in rapidity. The two-particle correlation function depends on $\cos (2 \Delta \phi)$ and higher order harmonics, where $\Delta \phi=\phi_{1}-\phi_{2}$ is the relative angle between two particles separated in rapidity.

An explanation of these phenomena was considered within the CGC framework for $\mathrm{p}+\mathrm{p}$ collisions in Ref. [8], and for $\mathrm{p}+\mathrm{Pb}$ collisions in Ref. [9]. Motivated by the convincing success in application to $\mathrm{A}+\mathrm{A}$ collisions, hydrodynamics has also been used for a description of $\mathrm{p}+\mathrm{Pb}$ collisions $[10,11,12,13]$. In hydrodynamic models, the dependence of the correlation function on $\cos (2 \Delta \phi)$ and $\cos (3 \Delta \phi)$ is naturally present owing to the elliptic and triangular flow. Thus assuming hydrodynamic evolution in $\mathrm{p}+\mathrm{Pb}$ collisions, the experimental data can be explained. It is also possible that only a joint description involving the CGC framework for the initial stage with subsequent hydrodynamic expansion would provide satisfactory agreement with the data.

Email addresses: abzdak@bnl.gov (Adam Bzdak), vskokov@bnl.gov (Vladimir Skokov) 
One way to distinguish between different models is to study the average transverse momentum of hadrons as a function of the number of produced particles. For example, it is known that hydrodynamic radial flow strongly affects average transverse momentum, $\left\langle p_{T}\right\rangle$, of protons but only slightly that of pions. Indeed, the momentum change due to the radial flow can roughly be estimated by $m \cdot v_{\text {flow }}$, where $m$ is the mass of the particle and $v_{\text {flow }}$ is the flow velocity. Thus careful analysis of $\mathrm{p}+\mathrm{A}$ data can elucidate to what degree hydrodynamics plays a role in $\mathrm{p}+\mathrm{A}$ collisions.

Recently the CMS Collaboration at the LHC revealed its preliminary data on the average transverse momentum of pions, kaons and protons as a function of produced particles in $\mathrm{p}+\mathrm{Pb}$ collisions at $\sqrt{s}=5.02 \mathrm{TeV}$ [14]. Interestingly, the average transverse momentum, $\left\langle p_{T}\right\rangle$, in $\mathrm{p}+\mathrm{Pb}$ collisions is quite different in comparison to $\mathrm{p}+\mathrm{p}$ collisions at similar energies. In both systems $\left\langle p_{T}\right\rangle$ is a rapidly growing function of the number of produced particles. Moreover, at a given number of particles $\left\langle p_{T}\right\rangle^{p A}<\left\langle p_{T}\right\rangle^{p p}$. All this features seem to be consistent with the CGC approach, as recently argued in Ref. [15], thus leaving very little room for the hydrodynamic evolution.

The data collected at the top RHIC and SPS energies suggest that $\mathrm{p}+\mathrm{A}$ collisions can be viewed, with a good precision, as a superposition of elementary $\mathrm{p}+\mathrm{p}$ collisions. This is strikingly manifested by the very good agreement of the $\mathrm{p}(\mathrm{d})+\mathrm{A}$ data with the prediction of the wounded nucleon model $[16,17]$

$$
N_{c h}^{p A}=N_{\text {part }} \frac{n_{c h}^{p p}}{2}
$$

where $N_{c h}^{p A}$ and $n_{c h}^{p p}$ are the average numbers of produced particles in $\mathrm{p}+\mathrm{A}$ and $\mathrm{p}+\mathrm{p}$ collisions, respectively, and $N_{\text {part }}$ is the number of wounded nucleons. The wounded nucleon model, and its modifications [18], proved very successful in understanding many features of heavy-ion collisions data. In this model, the interpretation of Eq. (1) is straightforward. In p+A collisions, a nucleon inside a nucleus is struck exactly once and subsequently produces a certain number of particles, on the average equal $n_{c h}^{p p} / 2$.

In this letter, we apply the wounded nucleon model to investigate how the average transverse momentum of particles in $\mathrm{p}+\mathrm{p}$ collisions reveals itself in $\mathrm{p}+\mathrm{A}$ collisions. ${ }^{1}$ Since $\left\langle p_{T}\right\rangle$ in $\mathrm{p}+\mathrm{p}$ collisions is a rapidly changing function of the number of produced particles [20], it is not clear how $\left\langle p_{T}\right\rangle$ in $\mathrm{p}+\mathrm{A}$ collisions, with many nucleon-nucleon interactions, depends on the number of produced particles. $^{2}$ Our calculations may serve as a natural baseline for an onset of collective physics (including CGC) irreducible to elementary nucleon-nucleon interactions.

\footnotetext{
${ }^{1}$ The question arises if the applicability of the wounded nucleon model to $\mathrm{p}+\mathrm{A}$ collisions breaks down at the LHC energies, where owing to the Lorentz contraction of the colliding nucleus, one could expect formation of coherent interacting color fields rather than a superposition of independent elementary nucleon-nucleon collisions [19].

${ }^{2}$ Only in the case $\left\langle p_{T}\right\rangle_{N}^{p p}=$ const, the prediction for $\mathrm{p}+\mathrm{A}$ collisions is trivial, that is, $\left\langle p_{T}\right\rangle_{N}^{p A}=$ const.
} 
Our main result is that the average transverse momentum in high multiplicity $\mathrm{p}+\mathrm{A}$ collisions weekly depends on the number of produced particles and underestimates the preliminary experimental data at the LHC. The difference is rather small for pions $(\sim 100 \mathrm{MeV})$ and greater for kaons $(\sim 300 \mathrm{MeV})$ and protons $(\sim 500 \mathrm{MeV})$. It is interesting that the magnitude of this difference is consistent with hydrodynamic expectations, where the radial flow pushes heavier particles to higher momenta.

In the next section, we formulate the problem analytically and present the results based on numerical calculations. The last section gives our comments and conclusions.

\section{Results and discussion}

Suppose we have $M$ independent sources (wounded nucleons) of particles in $\mathrm{p}+\mathrm{A}$ collisions. The single particle distribution for particle species $\alpha$ at a given transverse momentum, $p_{T}$, is obviously given by the sum of all sources ${ }^{3}$

$$
f_{\alpha}^{p A}\left(p_{T} ; N_{1}, \ldots, N_{M}\right)=\sum_{i=1}^{M} f_{\alpha}^{s}\left(p_{T} ; N_{i}\right),
$$

where $f_{\alpha}^{p A}\left(p_{T} ; N_{1}, \ldots, N_{M}\right)$ is the distribution function of particles $\alpha$ in $\mathrm{p}+\mathrm{A}$ collisions at a given total numbers of particles (number of tracks) $N_{i}$ coming from each source $i{ }^{4}$ The single-particle distribution function provides

$$
\int f_{\alpha}^{s}\left(p_{T} ; N_{i}\right) d^{2} p_{T}=\left\langle N^{\alpha}\right\rangle_{N_{i}}^{s}, \quad \int f_{\alpha}^{s}\left(p_{T} ; N_{i}\right) p_{T} d^{2} p_{T}=\left\langle p_{T}^{\alpha}\right\rangle_{N_{i}}^{s}\left\langle N^{\alpha}\right\rangle_{N_{i}}^{s},
$$

where $\left\langle N^{\alpha}\right\rangle_{N_{i}}^{s}$ is the average number of particles $\alpha$ from the $i$-th source at a given total number of particles, $N_{i}$, from this source; $\left\langle p_{T}^{\alpha}\right\rangle_{N_{i}}^{s}$ is the average transverse momentum.

Using Eq. (2), the average transverse momentum in $\mathrm{p}+\mathrm{A}$ collisions can be readily derived

$$
\left\langle p_{T}^{\alpha}\right\rangle_{N_{1}, \ldots, N_{M}}^{p A}=\frac{1}{\left\langle N^{\alpha}\right\rangle_{N_{1}, \ldots, N_{M}}^{p A}} \sum_{i=1}^{M}\left\langle p_{T}^{\alpha}\right\rangle_{N_{i}}^{s}\left\langle N^{\alpha}\right\rangle_{N_{i}}^{s},
$$

where we defined the total number of particles $\alpha$ (from all sources) by

$$
\left\langle N^{\alpha}\right\rangle_{N_{1}, \ldots, N_{M}}^{p A}=\sum_{i=1}^{M}\left\langle N^{\alpha}\right\rangle_{N_{i}}^{s} .
$$

\footnotetext{
${ }^{3}$ In this letter, we present calculations for positively charged pions, kaons, and protons; that is $\alpha=\pi^{+}, K^{+}, p$.

${ }^{4}$ Particles $\alpha$ and $N_{i}$ can, in principle, come from different rapidity or $p_{T}$ regions.
} 
It is natural to expect that mean number of particles $\alpha,\left\langle N^{\alpha}\right\rangle_{N_{i}}^{s}$, from one source is proportional to the total number of particles (or tracks) from a given source $N_{i}$, that is

$$
\left\langle N^{\alpha}\right\rangle_{N_{i}}^{s} \propto N_{i}
$$

This simplifies Eq. (4) into

$$
\left\langle p_{T}^{\alpha}\right\rangle_{N_{1}, \ldots, N_{M}}^{p A}=\sum_{i=1}^{M}\left\langle p_{T}^{\alpha}\right\rangle_{N_{i}}^{s} N_{i} / \sum_{i=1}^{M} N_{i} .
$$

The average transverse momentum in $\mathrm{p}+\mathrm{A}$ collisions at a given total number of produced particles $N_{\text {tot }}$ can be obtained by sampling distributions of $N_{i}$ such that $N_{\text {tot }}=\sum_{i} N_{i}$. In other words,

$$
\begin{aligned}
& \left\langle p_{T}^{\alpha}\right\rangle_{N_{\text {tot }}}^{p A}= \\
& \frac{1}{\mathcal{N}} \sum_{M=1}^{A+1} P_{G}(M) \sum_{N_{1}, \ldots, N_{M}} \frac{\sum_{i=1}^{M}\left\langle p_{T}^{\alpha}\right\rangle_{N_{i}}^{s}\left\langle N^{\alpha}\right\rangle_{N_{i}}^{s}}{\sum_{i=1}^{M}\left\langle N^{\alpha}\right\rangle_{N_{i}}^{s}} \prod_{i=1}^{M} P\left(N_{i}\right) \delta\left(\sum_{i=1}^{M} N_{i}-N_{\text {tot }}\right),
\end{aligned}
$$

where $P_{G}(M)$ is the distribution of the number of participants in $\mathrm{p}+\mathrm{A}$ collisions and $\mathcal{N}$ is the proper normalization given by

$$
\mathcal{N}=\sum_{M=1}^{A+1} P_{G}(M) \sum_{N_{1}, \ldots, N_{M}} \prod_{i=1}^{M} P\left(N_{i}\right) \delta\left(\sum_{i=1}^{M} N_{i}-N_{\text {tot }}\right) .
$$

In this paper, $P_{G}(M)$ is defined by the Glauber Monte Carlo simulations. The probability $P\left(N_{i}\right)$ is the multiplicity distribution of produced particles from the $i$-th source. Using Eq. (6) we obtain

$$
\begin{aligned}
& \left\langle p_{T}^{\alpha}\right\rangle_{N_{\text {tot }}}^{p A}= \\
& \frac{1}{\mathcal{N}} \sum_{M=1}^{A+1} P_{G}(M) \sum_{N_{1}, \ldots, N_{M}} \frac{\sum_{i=1}^{M}\left\langle p_{T}^{\alpha}\right\rangle_{N_{i}}^{s} N_{i}}{\sum_{i=1}^{M} N_{i}} \prod_{i=1}^{M} P\left(N_{i}\right) \delta\left(\sum_{i=1}^{M} N_{i}-N_{\text {tot }}\right) .
\end{aligned}
$$

To calculate $\left\langle p_{t}^{\alpha}\right\rangle_{N_{\text {tot }}}^{p A}$ we apply the Glauber Monte Carlo model. As usual we first sample positions of nucleons in a nucleus according to the Woods Saxon distribution with parameters given in Ref. [21]. The number of participants is then computed taking inelastic cross-section for $\mathrm{p}+\mathrm{p}$ collisions to be $\sigma=65$ $\mathrm{mb}[23] . .^{5}$ To each wounded nucleon we prescribed the number of produced particles, in $|\eta|<2.4$, sampled according to the negative binomial distribution ${ }^{6}$ with parameters obtained from experimental data [24], analysed in Ref. [25].

\footnotetext{
${ }^{5}$ We checked that hard-sphere and Gaussian inelastic profiles $[18,22]$ lead to the same results.

${ }^{6}$ Average number of particles and $k$ parameter in the negative binomial distribution equal to half of the values measured in $\mathrm{p}+\mathrm{p}$ collisions.
} 

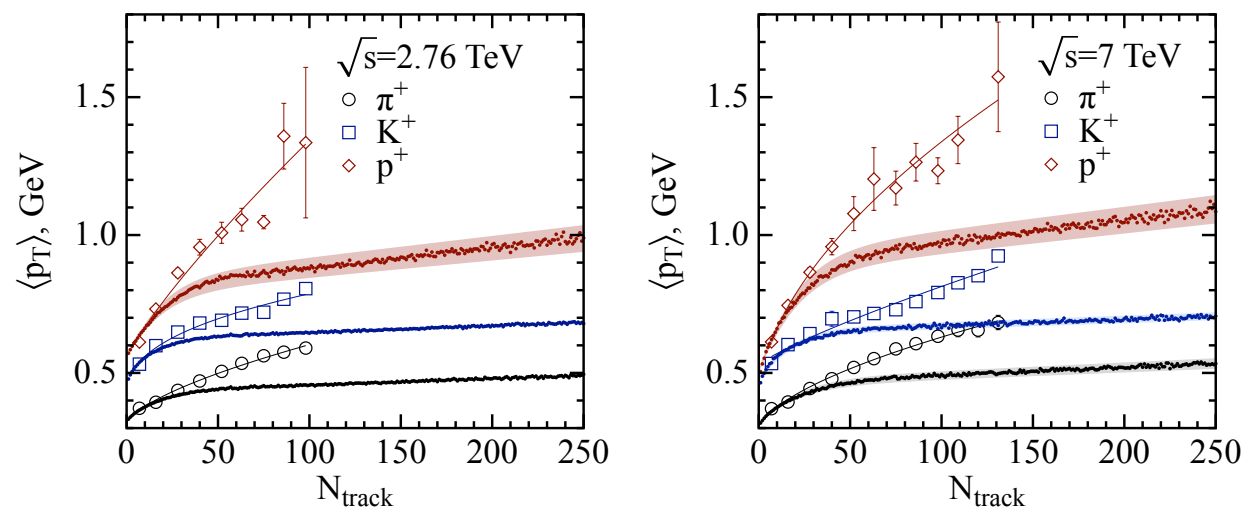

Figure 1: The average transverse momentum as a function of $N_{\text {track }}$, measured in $|\eta|<2.4$, for different particle species in the wounded nucleon model for $\mathrm{p}+\mathrm{Pb}$ collisions (small filled points) at $\sqrt{s}=2.76 \mathrm{TeV}$ and $7 \mathrm{TeV}$. The band shows theoretical uncertainty of the model due to variation of the coefficient $\kappa$ in Eq. (12) within the range [1.3,1.7]. The large open symbols are the experimental data on average transverse momentum in $\mathrm{p}+\mathrm{p}$ collisions, used as an input in our calculations.

In the case of $\mathrm{p}+\mathrm{p}$ collisions we have

$$
\left\langle p_{T}^{\alpha}\right\rangle_{N_{1}, N_{2}}^{p p}=\frac{\left\langle p_{T}^{\alpha}\right\rangle_{N_{1}}^{s} N_{1}+\left\langle p_{T}^{\alpha}\right\rangle_{N_{2}}^{s} N_{2}}{N_{1}+N_{2}} .
$$

If $N_{1}=N_{2}$ we obtain $\left\langle p_{T}^{\alpha}\right\rangle_{N_{1}, N_{1}}^{p p}=\left\langle p_{T}^{\alpha}\right\rangle_{N_{1}}^{s}$, if $N_{1} \gg N_{2}$ we get $\left\langle p_{T}^{\alpha}\right\rangle_{N_{1}, N_{2}}^{p p} \approx$ $\left\langle p_{T}^{\alpha}\right\rangle_{N_{1}}^{s}$. In the former case $\left\langle p_{T}^{\alpha}\right\rangle_{N_{\text {tot }}=2 N_{1}}^{p p}=\left\langle p_{T}^{\alpha}\right\rangle_{N_{1}}^{s}$ and the latter $\left\langle p_{T}^{\alpha}\right\rangle_{N_{\text {tot }} \approx N_{1}}^{p p} \approx$ $\left\langle p_{T}^{\alpha}\right\rangle_{N_{1}}^{s}$. We checked that for all practical purposes

$$
\left\langle p_{T}^{\alpha}\right\rangle_{N_{i}}^{s} \approx\left\langle p_{T}^{\alpha}\right\rangle_{N_{\mathrm{tot}}=\kappa N_{i}}^{p p}
$$

with $\kappa=1.5$. We use this approximation in our Monte Carlo calculations of $\mathrm{p}+\mathrm{A}$ collisions. The input for the average transverse momentum (12) in $\mathrm{p}+\mathrm{p}$ collisions is taken from the CMS data published in Ref. [20].

In Figure 1, we show the results for $\mathrm{p}+\mathrm{Pb}$ collisions obtained in the wounded nucleon model (small filled points) in comparison to $\mathrm{p}+\mathrm{p}$ experimental data (open symbols), which were used as an input in our calculations. We carried out the computations for two energies $\sqrt{s}=2.76 \mathrm{TeV}$ and $\sqrt{s}=7 \mathrm{TeV}$, for positively charged pions, kaons and protons. Figure 1 shows that $\mathrm{p}+\mathrm{Pb}$ calculations are always below experimental $\mathrm{p}+\mathrm{p}$ data points. This can be understood in the following simple way. In an ordinary $\mathrm{p}+\mathrm{A}$ collision the number of participants and consequently number of produced particles is larger than in an ordinary $\mathrm{p}+\mathrm{p}$ collision. Thus, comparing both systems at the same number of produced particles, say $N_{\text {track }}=100$, a typical nucleon-nucleon interaction in a p $+\mathrm{Pb}$ collision produces less than 100 particles. Consequently, owing to the non-flat dependence of average transverse momentum in $\mathrm{p}+\mathrm{p}$ as a function of $N_{\text {track}}$, 


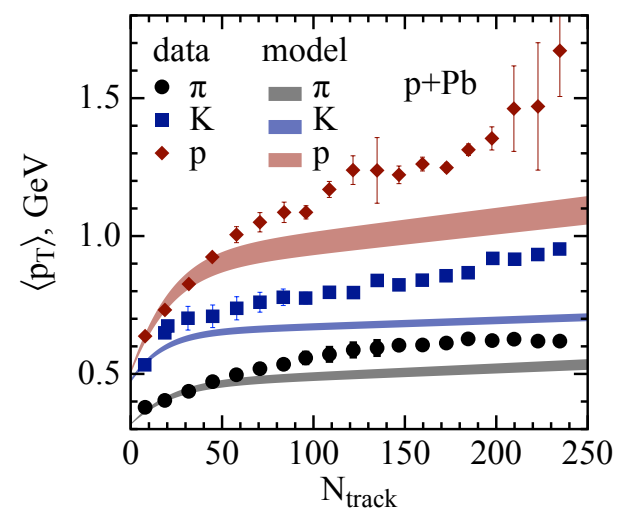

Figure 2: The average transverse momentum as a function of $N_{\text {track }}$ for protons, kaons and pions in the wounded nucleon model for $\mathrm{p}+\mathrm{Pb}$ collisions at $\sqrt{\mathrm{s}}=7 \mathrm{TeV}$ compared to the preliminary experimental data by the CMS Collaboration at $\sqrt{s}=5.02 \mathrm{TeV}$.

our $\mathrm{p}+\mathrm{Pb}$ results are below the $\mathrm{p}+\mathrm{p}$ data points. It is also worth noticing that our $\mathrm{p}+\mathrm{Pb}$ results are significantly flatter then the experimental $\mathrm{p}+\mathrm{p}$ data.

The CMS Collaboration has collected preliminary data in $\mathrm{p}+\mathrm{Pb}$ collisions at $\sqrt{s}=5.02 \mathrm{TeV}$ [14]. Since our model results do not change strongly in the energy range from $2.76 \mathrm{TeV}$ to $7 \mathrm{TeV}$, we will compare the preliminary $\mathrm{p}+\mathrm{Pb}$ experimental data with our model at $\sqrt{s}=7 \mathrm{TeV}$. As shown in Figure 2, our model results (bands) for protons, kaons and pions, are below the preliminary data (full symbols). For example, for $N_{\text {track }}=200$ our model calculations have a difference with experimental data of about $100 \mathrm{MeV}$ for pions, $300 \mathrm{MeV}$ for kaons and $500 \mathrm{MeV}$ for protons. First, this suggests that high multiplicity $\mathrm{p}+\mathrm{Pb}$ collision is irreducible to a superposition of $\mathrm{p}+\mathrm{p}$ collisions and that collective/coherent effects must be accounted for. Second, the apparent discrepancy between the preliminary experimental data and our results may be explained by some mechanism which shifts particles to higher momentum proportionally to their masses, as one expects in the hydrodynamic models, where, the shift in the transverse momentum is roughly proportional to $m \cdot v_{\text {flow }}{ }^{7}$ The final conclusion, however, requires quantitative analysis of the data in a hydrodynamic model.

\section{Comments and conclusions}

Using the wounded nucleon model, based on the available experimental data of $\mathrm{p}+\mathrm{p}$ collisions, we calculated the average transverse momentum of positively charged pions, kaons and protons in $\mathrm{p}+\mathrm{Pb}$ collisions. The model results show

\footnotetext{
${ }^{7}$ As seen in the mean transverse momentum difference between peripheral and central collisions in nucleus-nucleus collisions, see e.g., Ref. [26].
} 
that at a given number of particles (tracks) the average transverse momentum in $\mathrm{p}+\mathrm{Pb}$ collisions is significantly smaller then the one in $\mathrm{p}+\mathrm{p}$ collisions. We also compared our results with the preliminary CMS data in $\mathrm{p}+\mathrm{Pb}$ collisions and we observed that the wounded nucleon model underestimates the data. This demonstrates that in high multiplicity $\mathrm{p}+\mathrm{Pb}$ collisions at the LHC energy we encounter physics which cannot be reduced to a superposition of elementary $\mathrm{p}+\mathrm{p}$ collisions. The magnitude of the difference between our model results and the preliminary CMS data is consistent with hydrodynamic expectations, and is small for pions and greater for kaons and protons.

Finally we comment that similar studies in central $\mathrm{d}+\mathrm{Au}$ collisions would be very informative. ${ }^{8}$ In this case, Eq. (1) is experimentally verified to a good precision [17], that is, a $\mathrm{d}+\mathrm{Au}$ collision at $\sqrt{s}=200 \mathrm{GeV}$ can be viewed as a superposition of elementary nucleon-nucleon collisions, when the number of particles is considered. The possible hydrodynamic effects are not expected to change the number of particles. Thus, it would be useful to analyze the measured PHENIX data $[27,28]$ and extract the average transverse momentum in $\mathrm{p}+\mathrm{p}$ and $\mathrm{d}+\mathrm{Au}$ collisions at different centralities. If there is no radial flow, we expect to observe

$$
\left\langle p_{T}^{\alpha}\right\rangle^{d A u}=\left\langle p_{T}^{\alpha}\right\rangle^{p p}
$$

for all centralities and all particles species $\alpha$. Here $\left\langle p_{T}^{\alpha}\right\rangle^{p p}$ is averaged over all numbers of produced particles (minimum bias) and $\left\langle p_{T}^{\alpha}\right\rangle^{d A u}$ is averaged over all numbers of produced particles within a given centrality class. A possible radial flow in $\mathrm{d}+\mathrm{Au}$ collisions would increase $\left\langle p_{T}^{\alpha}\right\rangle^{d A u}$ so that

$$
\left\langle p_{T}^{\alpha}\right\rangle^{d A u}-\left\langle p_{T}^{\alpha}\right\rangle^{p p} \approx m_{\alpha} v_{\text {flow }}
$$

is small for pions and larger for kaons and protons.

\section{Acknowledgments}

We thank Larry McLerran, Robert Pisarski and Raju Venugopalan for stimulating discussions and constructive criticism. A.B. is supported through the RIKEN-BNL Research Center. V.S. is supported by the U.S. Department of Energy under contract \#DE-AC02-98CH10886.

\section{References}

[1] V. Khachatryan et al. [CMS Collaboration], JHEP 1009, 091 (2010).

[2] S. Chatrchyan et al. [CMS Collaboration], Phys. Lett. B 718, 795 (2013).

[3] B. Abelev et al. [ ALICE Collaboration], Phys. Lett. B 719, 29 (2013).

\footnotetext{
${ }^{8}$ We thank Anne Sickles for pointing this out to us.
} 
[4] G. Aad et al. [ATLAS Collaboration], Phys. Rev. Lett. 110, 182302 (2013).

[5] S. Chatrchyan et al. [CMS Collaboration], arXiv:1305.0609 [nucl-ex].

[6] S. Chatrchyan et al. [CMS Collaboration], JHEP 1107, 076 (2011).

[7] A. Adare et al. [PHENIX Collaboration], arXiv:1303.1794 [nucl-ex].

[8] K. Dusling and R. Venugopalan, Phys. Rev. D 87, 051502 (2013).

[9] K. Dusling and R. Venugopalan, Phys. Rev. D 87, 054014 (2013).

[10] P. Bozek, Phys. Rev. C 85, 014911 (2012).

[11] P. Bozek and W. Broniowski, Phys. Lett. B 718, 1557 (2013); Phys. Lett. B 720, 250 (2013); arXiv:1304.3044 [nucl-th].

[12] A. Bzdak, B. Schenke, P. Tribedy and R. Venugopalan, Phys. Rev. C 87, 064906 (2013).

[13] G. -Y. Qin and B. Mueller, arXiv:1306.3439 [nucl-th].

[14] F. Sikler for the CMS Collaboration, talk at the workshop on protonnucleus collisions at the LHC, ECT* Trento, Italy, 2013.

[15] L. McLerran, M. Praszalowicz and B. Schenke, arXiv:1306.2350 [hep-ph].

[16] A. Bialas, M. Bleszynski and W. Czyz, Nucl. Phys. B 111, 461 (1976).

[17] A. Bialas and W. Czyz, Acta Phys. Polon. B 36, 905 (2005).

[18] A. Bialas and A. Bzdak, Phys. Lett. B 649, 263 (2007); Acta Phys. Polon. B 38, 159 (2007); Phys. Rev. C 77, 034908 (2008).

[19] L. McLerran, private communication.

[20] S. Chatrchyan et al. [CMS Collaboration], Eur. Phys. J. C 72, 2164 (2012).

[21] B. Alver, M. Baker, C. Loizides and P. Steinberg, arXiv:0805.4411 [nucl-ex].

[22] M. Rybczynski and W. Broniowski, Phys. Rev. C 84, 064913 (2011).

[23] A. J. Zsigmond [CMS Collaboration], arXiv:1205.3142 [hep-ex].

[24] V. Khachatryan et al. [CMS Collaboration], JHEP 1101, 079 (2011).

[25] P. Ghosh, Phys. Rev. D 85, 054017 (2012).

[26] B. Abelev et al. [ALICE Collaboration], arXiv:1303.0737 [hep-ex].

[27] S. S. Adler et al. [PHENIX Collaboration], Phys. Rev. C 74, 024904 (2006).

[28] A. Adare et al. [PHENIX Collaboration], arXiv:1304.3410 [nucl-ex]. 